\title{
Quality control of tea products by variable sampling
}

\author{
Remzi Didmanidze*, and Nikolay Aldoshin \\ Russian Timiryazev State Agrarian University; Moscow, Russian Federation
}

\begin{abstract}
Based on the technological process of obtaining tea products, the factors determining its quality are considered. The analysis of these factors and their classification. The optimization of labor costs and time to perform quality control of raw materials using the methods of probability theory. Based on a simple discrete Markov chain, proposed a system of selective quality control of tea products by variable samples.
\end{abstract}

\section{Introduction}

One of the most consumed drinks in the world, tea, has become very popular. It is an excellent tonic that facilitates heart and vascular activity, lowers blood pressure, and increases vitality $[1,2]$.

Tea is the oldest drink known since before our era. It is produced in more than 30 countries on more than a million and a half hectares. The main producers of tea are Asian countries (India, China, Sri Lanka, etc.) [3-5].

In this article, we want to substantiate a system of quality control of tea products, ensuring the reduction of cost and time for its implementation.

\section{Methods}

The technological process of obtaining tea products is quite complicated and has a large labor intensity. Product quality depends on many factors of different nature (figure 1). All the variety of factors can be combined into several groups [6-12].

The first group can include factors associated with the care of tea plantations. These are, first of all, application of fertilizers for plant nutrition, pruning of tea bushes, control of pests and diseases, if necessary, irrigation [13, 14].

The second group of factors refers to the process of tea flush harvesting. The quality of the product, in this case, depends largely on the type of collection. Thus, higher quality tea can be obtained by manual collection than by machine collection. It should be noted that manual collection has much more labor-intensive work. It should also be noted that the quality of collection will depend on the timing and duration of their performance and the weather conditions in which these works are carried out $[15,16]$.

\footnotetext{
*Corresponding author: rdidmanidze@rgau-msha.ru
} 


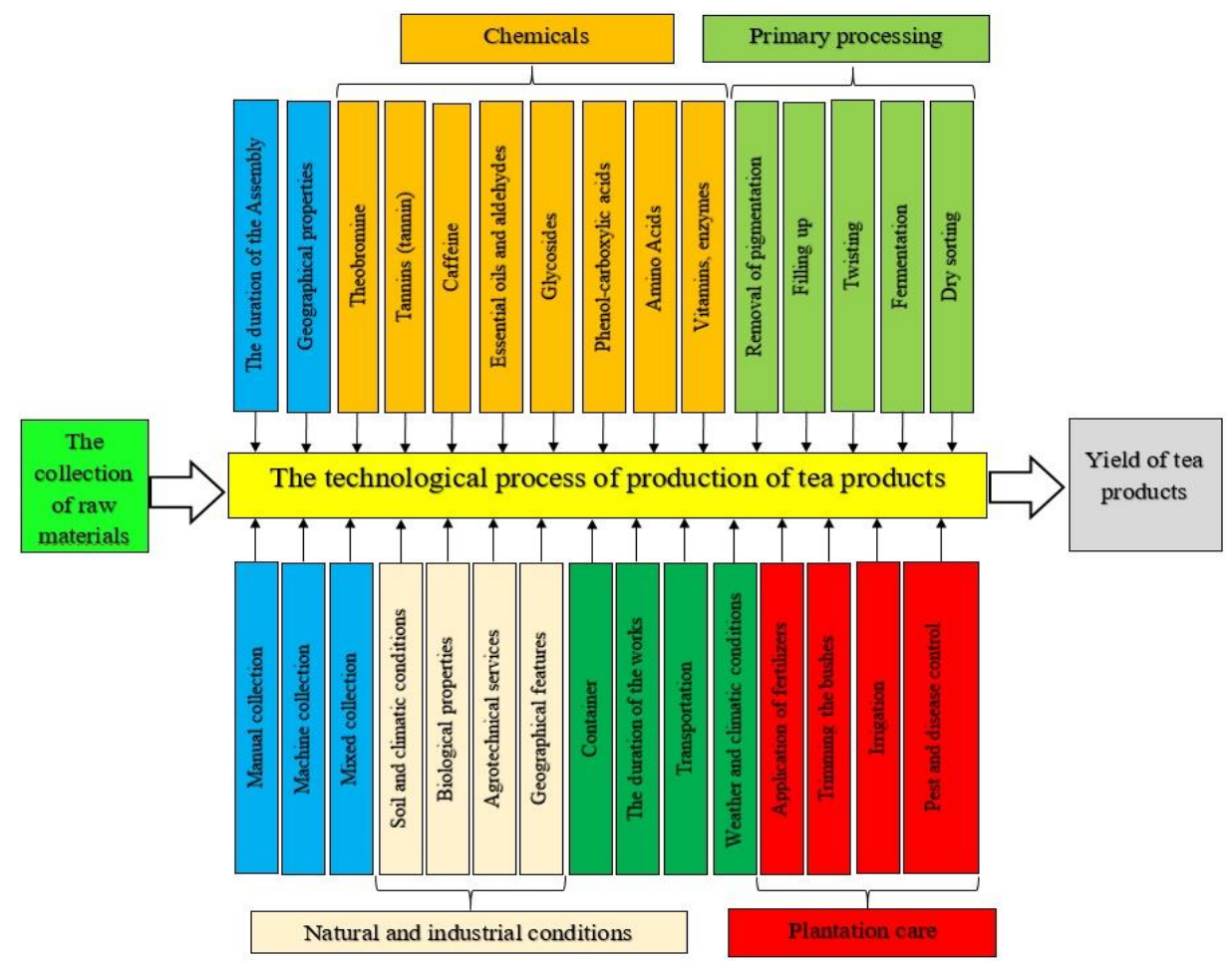

Fig. 1. Diagram of factors determining the quality of tea products

The third group is determined by a set of factors determining the transportation of harvested products on plantations to the places of their primary processing. The process of transportation is determined by the conditions of its implementation and timing. In this case, the conditions are determined by both the selected technology of transport works and natural-production conditions $[17,18]$.

The fourth group of factors forms the chemical composition of tea products. This may include many different indicators, which may change during the technological process of tea production. Dry substances determining the quality of tea raw materials can be conditionally divided into two parts: soluble in hot water and insoluble. The first part includes substances positively affecting the quality: phenolic compounds (tannin, catechins, flavone and anthocyanin glycosides, phenol carboxylic acids, etc.), essential oils and aldehydes, caffeine, theobromine and theophylline, amino acids, vitamins, enzymes, watersoluble carbohydrates, hydropectin, micro- and macroelements, etc. The second part includes ballast substances (substances that negatively affect quality): high-molecularweight polymers (cellulose, hemicellulose, lignin, protopectin, pectin acid), chlorophylls, insoluble proteins, etc. Most of them are involved in the creation of the cell membrane. The content of these substances in the mature bud and the first leaf is minimal and gradually increases in the second, third, fourth, etc. The high percentage of the mentioned substances in the tea leaf worsens the biochemical and technological properties of the raw material and makes it difficult to process. With the coarsening of tea raw materials, the content of substances of the first group gradually decreases, and the content of substances of the second group increases. In coarse tea leaves, the high content of dry matter is caused mainly by an increase in the number of ballast substances. The water-soluble fraction of the dry matter in tea technology is called extractive substances or extract. The insoluble fraction is ballast substances. Extractive substances determine the quality of raw tea. The 
content of extractive substances varies depending on the collection period, age of the leaf, its quality, etc. Delicate material contains more extractive substances than coarsened. In raw material production, their amount is $35-45 \%$ in terms of dry matter $[19,20]$.

The fifth group of factors is associated with the process of primary processing of tea leaves. Its content is determined by the performance of such operations as withering, twisting, fermentation, sorting, and, if necessary, removal of pigmentation of tea leaves.

Many factors determining the quality of tea products require detailed quality control during its production at different stages of the technological process. Such control requires a lot of labor and time, which can complicate and slow down the process of tea production.

When organizing the quality control of tea leaf preparation, there is a question of optimization of labor costs for these operations during the organizing the quality control of tea leaf preparation. Given the large volume of controlled material, the labor intensity of quality control is quite large. It requires a significant amount of time and labor, which prevents the rapid organization of work [21].

\section{Results and Discussion}

It is proposed to carry out quality control by variable volume, i.e., to evaluate small samples, and when the quality of the tea leaf to strengthen the control by increasing the volume of the sample. If the situation changes in the direction of increasing the quality of products, it is possible to return to the previous control method by small samples. This approach will optimize quality control, reducing the cost of its implementation while ensuring a sufficient level of quality of tea products. In this case, we have the so-called selective control.

To solve this problem, we will use the methods of probability theory. Control of not the entire batch of products, but only a relatively small batch, the so-called sample, allows us to conclude the quality of the product as a whole. The problem is to optimize the ratio between material damage from the probability of an increase in the share of low-quality products in small samples and the increase in time and labor costs in case of an increase in the volume of controlled products. Variable control allows making the process adjustable.

In a probabilistic sense, the test will be understood as a control operation to check the quality of the tea leaf during its preparation. Then, different variants of the outcome in such operations' performance can be considered a sequence of dependent tests.

In this formulation of the problem, we have a sequence of random events, as a result of which the considered system goes to different states: $A_{1}$ - control by a large sample, and $A_{2}$ - control by a small sample. If necessary, it is possible to set a different number of control levels. Transition to each subsequent state depends only on the previous one. In this connection, we can state that we have a random Markov process. In this case, it is a simple discrete Markov chain [2].

Sampling control will be carried out at certain periods as the next batch of tea products is accumulated. The process of determining the quality of tea leaf with variable control can be represented in the form of a stepped diagram (figure 2). For example, the control is carried out on a large sample at the first stage, then during three stages on a small sample, then again on a large sample, etc. 


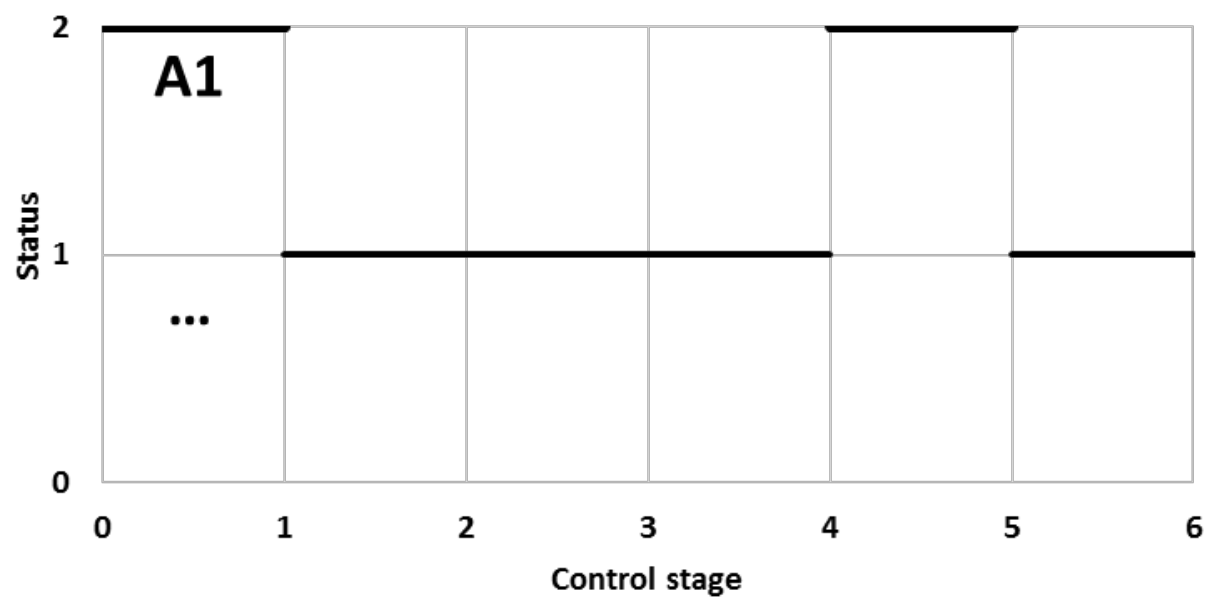

Fig. 2. Diagram of states by stages of quality control of tea products

The transition from one state to another depends on the probability of controlling a batch of tea products with large or small sample size and the amount of detected poor quality tea leaf in them. With the probability $P_{11}$, control will continue in a large sample and $P_{22}$ small. Then $P_{11}=1-P_{12}$ and $P_{22}=1-P_{21}$. This process can be described by the transition matrix, which has the following form:

$$
\Pi=\left[\begin{array}{cc}
1-\mathrm{P}_{12} & \mathrm{P}_{12} \\
\mathrm{P}_{21} & 1-\mathrm{P}_{21}
\end{array}\right]
$$

This matrix is stochastic since the sum of the numbers on the rows equals one, and all the elements are not negative. Also, there is no one among its values, indicating that there are no absorbing states. Inside the system (control process), you can get into any state. Since the set of states provides for the possibility of any transitions within the system from which one cannot exit, it allows us to assert that the process is ergodic.

Using the properties of ergodicity makes it easy to find the limiting (stationary) probabilities, which do not depend on the moment of choosing the starting point and the state of the quality control process. They are indicators of product control during the considered process as a whole.

The probabilities corresponding to large $\sigma_{1}$ or small $\sigma_{2}$ samples are found by determining the stationary matrix. The latter is determined $n$ by raising the transition matrix to the fifth power or from a system of linear equations using the normalization condition:

$$
\begin{gathered}
\sigma_{1}=\sigma_{1} \mathrm{P}_{11}+\sigma_{2} \mathrm{P}_{12} ; \\
\sigma_{2}=\sigma_{1} \mathrm{P}_{21}+\sigma_{2} \mathrm{P}_{22} ; \\
\sigma_{1}+\sigma_{2}=1 .
\end{gathered}
$$

Solving them concerning $\sigma_{1}$ and $\sigma_{2}$, we obtain:

$$
\begin{gathered}
\sigma_{1}=1-\mathrm{P}_{22} / 1+\mathrm{P}_{12}-\mathrm{P}_{22} ; \\
\sigma_{2}=\mathrm{P}_{12} / 1+\mathrm{P}_{12}-\mathrm{P}_{22} .
\end{gathered}
$$


The expected average number of incorrectly evaluated tea products, when controlled by samples $\mathrm{n}(i=1,2)$ of the batch, is equal:

$$
\begin{aligned}
& m\left(n_{1}\right)=x P_{12} \\
m\left(n_{2}\right)= & x P_{22}
\end{aligned}
$$

Where $x$ - is the number of incorrectly evaluated tea products.

Then the total volume of incorrectly monitored products will be

$$
M(x)=x P_{12} \sigma_{1}+x P_{22} \sigma_{2}
$$

The value obtained allows you to estimate the loss of quality of tea products when conducting sampling control by variable sampling.

\section{Conclusions}

This method allows you to reduce the cost of organizing the quality control of tea products, to estimate the number of improperly controlled products. The total labor intensity of the work is determined by the time of control of each sample.

\section{References}

1. Chandini S.K., Jaganmohan Rao L., Gowthaman M.K., Haware D.J., Sub- ramanian R. Enzymatic treatment to improve the quality of black tea extracts.- Food Chem. (2011), 127, № 3, pp 1039-1045.

2. Chen Huanwen, Liang Huazheng, Ding Jianhua, Lai Jinhu, Huan Yanfu, Qiao Xiaolin. Rapid differentiation of tea products by surface desorption atmospheric pressure chemical ionization mass spectrometry/- J. Agr. and Food Chem. - (2007), 55, № 25, pp 10093-10100.

3. Chen Su-yan, Deng Qin-lian, Wu Jing-jing, Chen Yan-hong. Application of ultrasonic wave in extracting tea - polyphenols/ Bohai daxue xuebao. Ziran kexue ban= J. Bohai Univ. Natur. Shi. Ed. - (2005), 26, № 4, pp 316-319.

4. Chen Xiao-giang, Ye Yang, Cheng Hao, Zhou Ying, Yang Ya-jun. Анализ спектров полисахаридов необработанного чая, выделенных различными методами- Guangpuxue yu guangpu fenxi=Spectrosc. And Spectral Anal. - (2009), 29, № 4, pp 1083-1087.

5. Ho, C. T., Lin, J. K. \& Shahidi, F. Tea and tea products: chemistry and health promoting properties. pp 1-283. CRC Press, (2008).

6. Lu, H. et al. Earliest tea as evidence for one branch of the Silk Road across the Tibetan Plateau. Sci. Rep. 6, 18955; doi: 10.1038/srep18955 (2016).

7. Gondoin Anals, Grussu Dominic, Stewart Derek, Mc Dougall Gordon J. While and green tea polyphenols inhibit pancreatic lipase in virto. 43, № 5, pp 1537 - 1544. (2010).

8. Zhang, J., Lu, H. \& Huang, L. Calciphytoliths (calcium oxalate crystals) analysis for the identification of decayed tea plants (Camellia sinensis L.). Sci. Rep. 4, 1-9, 10.1038/srep06703, (2014).

9. Karak, T. \& Bhagat, R. M. Trace elements in tea leaves, made tea and tea infusion: A review. Food Research International 43, pp 2234-2252, (2010). 
10. Fan, W., Gong, D., Yao, Z. \& Li, D. Identification analysis of carbonized suspected tea from Luan tomb in the northern song dynasty Agricultural archaeology 2, pp 212 217, (2012).

11. Krishnan Rajesh, Maru Girish B. Isolation and analyses of polymeric poly-phenol fractions from black tea/ Food Chem. - (2006), 94, № 3, pp 331-340.

12. Kule Janet A.M., Morrice Plilip C., McNeill Geraldine, Duthie Garry G. Effects of infusion time and addition of milk on content and absorption of polyphenols from black tea/ - J. Agr. and Food Chem. 55, № 12, pp 4889-4894. (2007).

13. Kumar Sangita D., Narayan G., Hassarajani S. Determination of anionic minerals in black and kombucha tea using ion chromatography/- Food Chem. 111, № 3, pp 784788. (2008).

14. Labbe David, Tremblay Angelo, Bazinct Laurent. Effect of brewing temperature and duration on green tea catechin solubilization: basis for production of EGC and EGCG-enriched fractions/- Separ. fnd Purif. 49, № 1, pp 1-9. (2006).

15. Lee Jang-Eun, Lee Bum-Jin, Chung Jin-Oh, Shin Ilyun-Jung, Lee Sang-Jun, Lee Cherl-Ho, Hong Young-Shick. H NMR-based metabolomic characterization during green tea fermentation - Food Res. 44, № 2, pp 597-604. (2011).

16. Lee S., Park M.K., Kim K.H., Kim Y.S. Effect of supercritical carbon dioxide decaffeination on volatile components of green teas/- J. Food Sci. 72, № 7, pp S497S502. (2007).

17. Li Ping, Wang Yanhui, Ma Runyu, Zhang Xiaolin. Separation of tea polyphenol in Green Tea Leaves by a combined CATUFM-adsorption resin process/- J. Food Eng. 67, № 3, pp 253-260. (2005).

18. Lu You, Yang Xingbin, Zhao Yan, Ruan Yun, Yang Ying, Wang Zhezhi. Separation and quantification of component monosaccharides of the tea polysaccharides from Gynostemma pentaphyllum by HPLC with indirect UV detection/- Food Chem. 112, № 3, pp 742-746. (2009).

19. Mizukami Yuzo, Sawai Yusuke, Yamagushi Yuichi. Changes in concentrations of acrylamide, selected odorants, and catechins caused by roas-ing of green tea/- J. Agr. and Food Chem. 56, № 6, pp 2154- 2159. (2008).

20. Deng, W. W., Ogita, S. \& Ashihara, H. Distribution and biosynthesis of theanine in Theaceae plants. Plant Physiology and Biochemistry 48, pp 70-72, (2010).

21. Muthumani Thomas, Kumar Senthil R.S. Influence of fermentation time on the development of compounds responsible for quality in black tea/- Food Chem. 101, № 1, pp 98-102. (2007). 